\title{
HOGARES LIDERADOS POR MUJERES Y POR HOMBRES: EL USO DEL TIEMPO Y LAS DESIGUALDADES
}

\author{
HOUSEHOLDS HEADED BY WOMEN AND MEN: TIME USE AND \\ INEQUALITIES
}

\section{FAMÍLIAS CHEFIADAS POR MULHERES E POR HOMENS: USO DO TEMPO E DESIGUALDADES}

\section{Marisa Bordón Ojeda ${ }^{1}$}

\begin{abstract}
Resumen
Este trabajo tiene como objetivo constatar que existe una situación de desigualdad económica y social entre hogares liderados por mujeres y aquellos liderados por hombres en Costa Rica. Para ello, se tendrán en cuenta las variables del tiempo que en ambos hogares dedican a las diferentes actividades y los ingresos que perciben los jefes y jefas de hogar, a partir de los datos de la muestra obtenidos en la Encuesta Nacional del Uso del Tiempo 2017. Para el análisis, se utilizaron los datos promedio obtenidos en la encuesta para el colectivo de jefes y jefas de familia correspondientes a las variables de la dedicación de su tiempo y los ingresos. Como resultado del análisis, se verifican diferencias en el tiempo que los jefes y jefas dedican al trabajo no remunerado y al trabajo remunerado, así como diferencias en la remuneración que reciben por su actividad principal. Estas evidencias, nos llevan a reflexionar sobre la necesidad de implementar políticas públicas que compensen la situación desigual que padecen los hogares liderados por mujeres.
\end{abstract}

Palabras claves: Jefas de hogar, desigualdad, corresponsabilidad social de los cuidados, división sexual del trabajo, perspectiva feminista.

\begin{abstract}
This paper aims to confirm economic and social inequality between households headed by women versus by men in Costa Rica. For this, the variables of time dedicated to different activities and income received by both heads of households will be taken into account, based on sample
\end{abstract}

Doi: https://doi.org/10.15359/eys.26-59.3

Recibido: 04-03-2021. Reenvíos: 21-04-2021. Aceptado: 14-05-2021. Publicado: 21-04-2021.

1 Doctora en Economía, Universidad Complutense de Madrid, España. Correo: marisaleonorbordon@ucm. es, ORCID: https://orcid.org/0000-0002-7577-0195

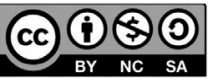


data obtained in the National Survey of Time Use 2017. Average data of variables of the heads of household time and income were used in the analysis. As a result, differences are verified between the time dedicated to unpaid and paid work by male and female heads of household, as well as differences between the remuneration received for their main activity. This evidence leads us to reflect on the need to implement public policies that compensate for the unequal situation suffered by households headed by women.

Keywords: female heads of household, inequality, social co-responsibility of care, sexual division of labor, feminist perspective.

\section{Resumo}

Este trabalho tem como objetivo evidenciar a existência de desigualdade econômica e social entre os domicílios chefiados por mulheres e aqueles chefiados por homens na Costa Rica. Para tanto, serão consideradas as variáveis do tempo que ambos os domicílios dedicam a atividades distintas e a renda recebida pelos chefes de família, com base nos dados da amostra obtida na Pesquisa Nacional de Uso do Tempo 2017. Para a análise, foi utilizada a média dos dados obtidos na pesquisa para o grupo de chefes de família correspondentes às variáveis de dedicação de seu tempo e renda. Como resultado da análise, encontram-se diferenças no tempo em que homens e mulheres chefes de família se dedicam ao trabalho não remunerado e ao remunerado, bem como diferenças na remuneração que recebem pela atividade principal. Essas evidências nos levam a refletir sobre a necessidade de implementação de políticas públicas que compensem a situação de desigualdade vivida pelos domicílios chefiados por mulheres.

Palavras-chave: mulheres chefes de família, desigualdade, corresponsabilidade social pelo cuidado, divisão sexual do trabalho, perspectiva feminista.

\section{Introducción}

En este trabajo se realiza el análisis de los resultados obtenidos en la Encuesta Nacional del Uso del Tiempo 2017 (ENUT, 2017), con el fin de observar las diferencias entre hogares costarricenses liderados por hombres y mujeres. Además, dentro de este colectivo se han analizado concretamente los casos de mujeres que lideran el hogar solas. Las variables analizadas incluyen ingresos y dedicación a las actividades remuneradas y no remuneradas. El objetivo es constatar, mediante la revisión de estos datos, la existencia de desigualdades socioeconómicas entre jefas y jefes, lo que afectará también al resto de miembros del hogar.

En concreto, se compararon variables en el ámbito de lo mercantil, como la remuneración que ambos colectivos percibieron por su ocupación principal y secundaria, la posición 
que ocupan en su actividad principal y la media de horas semanales dedicadas a las tareas remuneradas. Para el análisis del ámbito de las tareas fuera del mercado se ha utilizado, como variable, la media de las horas semanales dedicadas a las tareas no remuneradas de variada tipología.

El análisis que se realiza en este trabajo arroja luz sobre la situación que padecen las mujeres que lideran hogares, desde la perspectiva de los ingresos y de las horas de trabajo que dedican a las tareas, tanto en el ámbito de los mercados como fuera de estos.

Los datos analizados corresponden a la muestra de 4560 viviendas y 11400 personas aproximadamente, es decir, no se ha realizado con ellos los cálculos estadísticos necesarios para realizar las estimaciones a nivel nacional, como si se ha hecho con los datos publicados oficialmente ${ }^{2}$. Por lo tanto, las conclusiones que se extraen del análisis nos dan indicios de cuál puede ser la situación a nivel nacional, aunque debe tenerse en cuenta que pueden existir errores de sesgo, pese a la representatividad de la muestra.

Para abordar el análisis, en el apartado 2, se realiza una síntesis de algunos conceptos teóricos que han guiado el estudio, y en el apartado 3, se describe brevemente el contexto de las variables analizadas, teniendo en cuenta las condiciones del mercado laboral, las características de los hogares con jefas a la cabeza y las características de los hogares monoparentales en Costa Rica.

En el apartado cuarto se incluye el estudio empírico de la situación de las jefas y jefes en el ámbito del mercado laboral y en el quinto se tratan los datos que reflejan la situación en el ámbito de las tareas no remuneradas.

Por último, se realizan algunas reflexiones finales, donde se intenta incorporar algunas ideas sobre el papel potencial de las políticas públicas tanto en el ámbito laboral como en lo referente a las políticas de igualdad.

\section{Enfoques desde la teoría}

En el presente trabajo hemos optado por tomar como objeto de estudio las jefas y jefes de hogares. Para ello hemos tomado la definición de la encuesta, además de una categorización que refuerza los conceptos de jefe y jefa de hogar, realizando una diferenciación respecto del hogar monoparental, con el fin de resaltar la importancia de analizar la situación de este colectivo de forma separada.

En la ENUT (2017) se define al jefe o jefa de hogar como la persona o personas consideradas en la jefatura por las demás personas del hogar. En los grupos familiares la jefatura la ostenta quien tiene la autoridad máxima, quien lleva la administración del hogar, quien tiene más tiempo de residir ahí o quien posee más edad. Estos criterios también resultan válidos si el sujeto informante de un hogar que constituye un grupo

2 Se puede consultar la metodología en "Encuesta Nacional de Uso del Tiempo 2017. Resultados Generales". https://www.inec.go.cr/sites/default/files/documetos-biblioteca-virtual/reenut2017.pdf 
familiar no logra señalar expresamente cuál es la persona jefa de dicho hogar. En el caso de una persona que vive sola, ella misma es la jefatura del hogar.

Budlener (2003) establece tres criterios que pueden contribuir a ampliar la comprensión de este concepto. El primero es el criterio de autodesignación. El segundo es el criterio de autoridad, es decir, aquella persona que se identifica como una autoridad, es quien controla los recursos para mantener el hogar e impone las reglas y toma las decisiones importantes. Por último, se incorpora el criterio económico, que se expresa, a su vez, en otros dos criterios: la provisión principal de recursos al hogar y la tenencia de la vivienda (propietaria o arrendataria).

La definición tradicional de monoparentalidades es una estructura familiar integrada por un o una progenitora y su progenie (Barrón, 2002), y la evidencia empírica muestra que, en su mayoría, sus hogares son liderados por mujeres ${ }^{3}$.

El objetivo del trabajo es evidenciar las diferencias existentes entre los colectivos de jefas y jefes de familia. Para ello se optó por realizar el análisis de estas diferencias desde la perspectiva feminista de la economía. Esta corriente interpreta que las actividades económicas son los procesos de aprovisionamiento social, incluidos aquellos que no pasan por el mercado. Además, entienden que las relaciones de género son parte constitutiva del sistema socioeconómico, y los análisis de la realidad que se llevan a cabo buscan, como fin, la implementación de políticas (Pérez, 2014).

Desde esta perspectiva, se buscará constatar la existencia de relaciones económicas que sugieren desigualdad entre hombres y mujeres, tanto dentro del mercado como fuera de este. Como afirma Amaia Pérez Orozco (2014: 46), la economía feminista busca revelar lo oculto, aquellas tareas que no pasan por los mercados y cuya "visibilización muestra que las mujeres que no están en el mercado laboral, bien lejos de estar inactiva, están muy presentes en la economía".

Ambos trabajos, el que pasa por el mercado y el que permanece oculto, impulsan el ansiado crecimiento económico. Por lo tanto, ambos trabajos deberían tenerse en cuenta a la hora de calcular la actividad económica de una determinada sociedad. Pese a la posibilidad de contabilizar esta aportación en horas, existe un componente en las tareas ocultas que no puede materializarse en datos numéricos, lo que hace su aportación mucho más importante que el simple impulso de la producción. Cristina Carrasco (2013) afirma que este trabajo incluye aspectos que no pueden ser comprados en el mercado que tienen que ver con lo emocional, la socialización y el cuidado de la salud.

Pero las dificultades, como comentábamos unas líneas más arriba, se encuentran en el cálculo de la contribución de estas actividades. Las tareas domésticas y de cuidados que se realizan en el interior de los hogares y el trabajo remunerado son interdependientes, y persiguen objetivos diferentes. El primero tiene como fin el cuidado de la vida de las personas que integran el hogar, mientras que las tareas en el mercado de trabajo

3 Véase Barrientos, et. al., 2013 
forman parte del proceso productivo cuyo fin es la obtención de un beneficio. El trabajo no remunerado conlleva no solo el aporte emocional, sino también una serie de consecuencias en las mujeres, que impactan en su posición desigual frente al hombre. Las mujeres no solo organizan su vida entre las necesidades humanas y el trabajo en el mercado, sino que además el trabajo doméstico y de cuidados que ellas realizan permiten a los hombres participar en el mercado laboral sin restricciones (Carrasco, 2013).

En el ámbito del mercado, las relaciones de subordinación/dominación entre sexos, que se sitúan en las relaciones familiares patriarcales, afectan la naturaleza estructural de los puestos de trabajo. Así, la división sexual del trabajo se explica por la asignación a la mujer de las tareas asociadas a la reproducción de la fuerza de trabajo (cuidados y mantenimiento de la familia), que derivan de la reproducción biológica, pero que en realidad no corresponden con una asignación natural, sino social (Benería, 1981) ${ }^{4}$.

El capital se apropia de la fuerza de trabajo en los hogares por debajo de su valor (su coste, que pasa a formar parte de la acumulación de capital, invisibilizando así la gran cantidad de trabajo realizado por las mujeres (Carrasco, 2017).

La consideración de las tareas no domésticas como naturales de los hombres y las domésticas de las mujeres subyace en el menor valor de los trabajos que realizan las mujeres en el mercado laboral y en su discriminación salarial. La valoración social que se da a las tareas asociadas al sexo limita el acceso de las mujeres a los trabajos más cualificados (Brunet y Santamaría, 2016). Según esta división sexual del trabajo, se asocia a la feminidad los trabajos con menos poder socioeconómico (Pérez, 2014).

Como resultado de la incorporación de las mujeres al trabajo remunerado se intensifica su carga global de trabajo (remunerado y no remunerado) y, con ello, se reduce su tiempo de ocio, recreación y cuidados personales (desarrollo personal) (Carrasco, 2001). Son ellas las que asumen el coste de esta inserción a través de servicios de cuidado adquiridos en el mercado o requieren políticas públicas y redes familiares.

La dedicación de las mujeres al trabajo doméstico y de cuidados limita su participación en el mercado laboral en condiciones de igualdad con los hombres, lo cual lleva a las diferencias de salarios y pensiones en el futuro. No obstante, la Organización Internacional del Trabajo (OIT) señala que estas desigualdades no solo se deben a que las mujeres trabajan menos horas para poder conciliar el trabajo fuera y dentro del hogar, sino que existen otros factores explicativos. Entre ellos encontramos la dificultad para ocupar altos cargos y la infravaloración de su trabajo en ocupaciones y empresas altamente feminizadas (OIT, 2019a). El "Informe mundial sobre salarios 2018/2019" afirma que "las mujeres suelen obtener un rendimiento salarial menor de su educación que los hombres, aunque tengan la misma categoría ocupacional" (OIT, 2019b: 6).

4 La división sexual del trabajo está acompañada de una división étnica y de clase, ya que la identidad de las personas es un proceso múltiple (Brunet y Santamaría, 2016). 
Los hombres reciben ingresos laborales más altos que las mujeres por cada hora trabajada en la ocupación principal, esta diferencia se amplía en los colectivos de ingresos más bajos, entre los que se encontrarían los hogares monoparentales (OIT, 2019a: 58).

Más allá de las diferencias en el mercado laboral, la explicación de esta situación desigual es más profunda. El trasfondo es la división sexual del trabajo que se ha reproducido a través del tiempo, que desde los orígenes del sistema capitalista ubica a la mujer en la reproducción de la fuerza de trabajo, la excluye del mercado laboral y la somete a los hombres $^{5}$. La continuidad de la posición de las mujeres en el sistema económico impide alcanzar una situación de igualdad en lo laboral (salarios, puestos de trabajo, pensiones, etc.) con sus consecuencias en términos de mayor riesgo de pobreza y, en lo personal y emocional, tener menos tiempo para su desarrollo personal, como resultado de una mayor dedicación de horas al trabajo remunerado y no remunerado.

\section{Situación de los hogares monoparentales en Costa Rica}

Los datos de la Encuesta Nacional de Hogares (ENAHO) de 2017 muestran que el 38,3 $\%$ de los hogares tienen una jefatura femenina, mientras que el $28,9 \%$ de las jefas son mujeres sin pareja. Es decir, que el $74,3 \%$ de los hogares con jefatura femenina, solo tienen a la mujer a la cabeza del hogar.

Según el informe realizado del Censo Nacional de Población y Vivienda en 2011, las familias monoparentales representaban el 20,7 \% de los hogares del país (Barrientos et al., 2013), por lo que el porcentaje de hogares liderados por madres solteras con hijos o hijas habría aumentado respecto al censo anterior. En este estudio se afirma también que el $87,6 \%$ de los hogares monoparentales tienen a mujeres en la jefatura del hogar.

Entre las razones que se ofrecen para el incremento de hogares monoparentales, el estudio menciona que ante la separación o divorcio son las mujeres las que se hacen cargo de su parentela y mantienen su hogar, hecho que asocia a los roles tradicionales de hombres y mujeres presente en el "discurso oficial" que mantienen y reproducen los grupos de poder. El $42,8 \%$ de los hogares compuestos por madre y sus proles, corresponde a mujeres que se han separado o divorciado, mientras que el $29 \%$ son solteras (Barrientos et al., 2013).

El nivel de educación tiene su reflejo en las ocupaciones de que tienen las jefas de hogar: el 48,3\% de los hogares monoparentales solo alcanzan el nivel primario de educación, el $29,5 \%$ de las jefas están dedicadas a ocupaciones elementales ${ }^{6}$ y el $18,5 \%$ a la venta en locales y servicio directo, puestos de trabajo que no requieren especialización ni formación académica avanzada (Barrientos, et al., 2013).

5 Véase Federici (2004).

6 Según la Clasificación Internacional Uniforme de Ocupaciones (CIUO) utilizada en el Censo de Población y Vivienda de 2011, las ocupaciones elementales incluyen limpiadores y asistentes, peones agropecuarios, pesqueros y forestales, peones de minería, la construcción, la industria manufacturera y el transporte, ayudantes de preparación de alimentos, vendedores ambulantes de servicios y afines, recolectores de desechos y otras ocupaciones elementales. 
Según datos del Instituto Nacional de Estadísticas y Censos de Costa Rica (INEC, 2017) los ingresos percibidos por las mujeres en su empleo principal han sido 13,3\% menores a los de los hombres. El desempleo afecta en mayor medida a las mujeres, la tasa del desempleo en 2017 fue 11,6 \% frente al 7,5\% para los hombres (OIT, 2019a). Los hogares liderados por mujeres se encuentran en una situación de mayor vulnerabilidad y refleja en los niveles de pobreza: el $44,8 \%$ de los hogares pobres son hogares con una jefatura femenina, según la ENAHO de 2017.

Por último, las mujeres costarricenses dedican 41,2 horas por semana a las tareas no remuneradas ( $74,4 \%$ del conjunto de las horas dedicadas a estas tareas) frente a las 14,2 horas que dedican los hombres (25\%) (OIT, 2019a).

\section{El ámbito del mercado}

Según los datos de la Encuesta Nacional del Uso del Tiempo 2017, el porcentaje de jefas de hogares es el $39,42 \%$, frente al $60,58 \%$ de jefes.

Las variables se han analizado también para los hogares monoparentales, es decir, aquellos hogares en donde existe una sola madre o padre al frente del hogar.

Si bien existe una variedad de situaciones de monoparentalidad, hemos tomado bajo este concepto a las personas divorciadas, separadas, viudas y solteras. Con la intensión de reconocer las diferencias entre los casos mencionados, intentaremos matizar la situación de las mujeres divorciadas, que tienen una protección legal mayor y, por ello, una corresponsabilidad económica y de cuidado. Del particular, es preciso aclarar que no hemos podido separar los hogares que tienen jefas divorciadas, separadas, viudas y solteras en los que vive una única persona de las que conforman un núcleo familiar. Asumimos que, en estas familias, nuestras variables estarán afectadas por este problema.

El $60,81 \%$ del conjunto de jefas y jefes divorciados son mujeres, mientras que en situación de separación el porcentaje de mujeres se dispara al 78,61\%. El 81,77\% de las jefaturas en situación de viudedad son mujeres, se suaviza la proporción en el caso de las solteras, con un $52,23 \%$ de jefas.

Como mencionamos en el primer apartado, ser jefa o jefe de hogar implica entre otras responsabilidades la aportación principal de recursos económicos. Teniendo en cuenta que este es uno de los criterios que define a una jefa o jefe de hogar, esta será una de las variables que nos permitirá comprobar cuál es la situación de las mujeres al frente de hogares, en términos relativos. Además de los ingresos y posición que tienen en su ocupación principal, tendremos en cuenta también las horas que dedican al trabajo remunerado.

La variable ingresos nos dará la perspectiva monetaria de los niveles de desigualdad, mientras que la posición en el trabajo contribuirá a caracterizar la situación laboral. El acceso limitado a altos cargos es uno de los factores que explican la brecha salarial y una característica no monetaria (cualitativa) de la desigualdad. 
La cantidad de horas trabajadas remuneradas y las horas dedicadas al trabajo invisibilizado nos dará una perspectiva más amplia sobre los niveles de desigualdad. En la órbita del trabajo no remunerado se reproducen y acentúan las condiciones de desigualdad que se dan en el mercado entre hombres y mujeres.

En el caso de los ingresos provenientes de la ocupación principal, los hombres ganan una media de 519 763,32 colones, la media de las mujeres es de 318 586,9 colones. Es decir, las jefas ganan un $61 \%$ de lo que gana los jefes en su ocupación principal, lo que confirma la existencia de la conocida "brecha salarial"7. Matizamos esta información con los datos para las jefas que están solas frente al hogar. En la Tabla 1, las mujeres divorciadas y separadas tienen un ingreso por encima de la media del conjunto de las jefas, mientras que las mujeres viudas y solteras tienen un ingreso medio que está por debajo de la media general. Este matiz nos sugiere que existe un colectivo dentro de las jefas en una situación de mayor vulnerabilidad.

\section{Tabla 1}

Ingresos medios por ocupación principal y secundaria jefas hogares monoparentales (colones)

\begin{tabular}{lllll}
\hline & $\begin{array}{l}\text { Jefas } \\
\text { solteras }\end{array}$ & $\begin{array}{l}\text { Jefas } \\
\text { viudas }\end{array}$ & $\begin{array}{l}\text { Jefas } \\
\text { divorciadas }\end{array}$ & $\begin{array}{l}\text { Jefas } \\
\text { separadas }\end{array}$ \\
\hline Ocupación principal & $281.379,42$ & $307.069,92$ & $321.834,13$ & $363.175,19$ \\
Ocupación secundaria & $43.134,93$ & - & $102.595,64$ & $39.623,60$ \\
\hline
\end{tabular}

Nota: Elaborado con base en datos de la ENUT, 2017.

En cuanto a los ingresos que provienen de ocupaciones secundarias, los jefes perciben en promedio 188 110,33 colones, frente a 60 769,74 colones que perciben las jefas. Así mismo, si observamos los datos de la Tabla 1, correspondientes a los ingresos por la ocupación secundaria, vemos datos por debajo de la media general, excepto en el colectivo de las mujeres divorciadas. Una de las explicaciones plausibles es que, al compartir responsabilidades con sus excónyuges, pueden dedicar más tiempo a estas tareas y, por ello, obtener un mayor ingreso.

Una variable que puede aportar un matiz adicional a los ingresos es la posición de los jefes y jefas en su ocupación principal. Hay un 0,22 \% de mujeres y un 1,64 \% de hombres al frente del hogar que se desempeñan como directoras o gerentes. En el caso de las familias monoparentales, no hay mujeres que desempeñen este puesto.

Los datos mejoran en las profesionales científicas e intelectuales, con porcentajes similares ( $8,57 \%$ hombres y $8,63 \%$ mujeres), mientras que, en la tercera mejor posición laboral, la de técnicos y profesionales de nivel medio hay un $11,56 \%$ de jefes y un $2,79 \%$ de jefas. En la Tabla 2 se aportan los datos para los hogares con una mujer sola al frente.

7 Se define como brecha salarial la diferencia que existe entre los salarios percibidos por hombres y mujeres. 


\section{Tabla 2}

Posición en la ocupación principal jefas de hogares monoparentales

\begin{tabular}{|c|c|c|c|c|}
\hline \multirow{2}{*}{ Posición en trabajo principal } & $\begin{array}{l}\text { Jefas } \\
\text { solteras }\end{array}$ & Jefas viudas & $\begin{array}{l}\text { Jefas } \\
\text { divorciadas }\end{array}$ & $\begin{array}{l}\text { Jefas } \\
\text { separadas }\end{array}$ \\
\hline & \multicolumn{4}{|c|}{ En porcentajes } \\
\hline Director y gerentes & 0,00 & 0,00 & 0,00 & 0,00 \\
\hline Profesionales científicos e intelectuales & 9,29 & 3,82 & 12,85 & 4,45 \\
\hline Técnicos y profesionales de nivel medio & 0,99 & 1,11 & 6,64 & 2,71 \\
\hline
\end{tabular}

Nota: Elaborado con base en datos de la ENUT 2017.

Si bien, como comentábamos más arriba, no hay en este colectivo mujeres directoras y gerentes, las jefas solteras y divorciadas mejoran su porcentaje en el desempeño de profesiones científicas e intelectuales, mientras que las viudas y las separadas se ubican por debajo del porcentaje general de las jefas.

Los datos hasta ahora analizados no solo constatan la situación de desigualdad en los ingresos entre hombres y mujeres que lideran el hogar, sino que, además, confirman la existencia de mayor desigualdad en algunos colectivos monoparentales. Por otro lado, la existencia de límites para llegar a las posiciones más altas de la vida profesional también queda demostrada con los datos aportados por la ENUT 2017. Estas diferencias en el ámbito de los mercados afectarán sus salarios, pero también, en el futuro, el cálculo de sus pensiones, lo cual reproduce y a amplía la condición de inequidad.

La Tabla 3 muestra que los hombres a cargo de hogares tienen una dedicación mayor a las ocupaciones remuneradas, tanto en la principal (13 horas más) como en la secundaria (el doble), que las mujeres en su misma situación.

El grupo de mujeres viudas y solteras está por debajo del promedio general de las mujeres.

\section{Tabla 3}

Horas semanales dedicadas a tareas remuneradas (media)

\begin{tabular}{lcccccc}
\hline & Jefes & Jefas & $\begin{array}{l}\text { Jefas } \\
\text { solteras }\end{array}$ & $\begin{array}{l}\text { Jefas } \\
\text { viudas }\end{array}$ & $\begin{array}{l}\text { Jefas } \\
\text { divorciadas }\end{array}$ & $\begin{array}{l}\text { Jefas } \\
\text { separadas }\end{array}$ \\
\hline $\begin{array}{l}\text { Horas dedicadas a la } \\
\text { ocupación principal }\end{array}$ & 46,4 & 33,52 & 33,11 & 27,7 & 35,47 & 36,02 \\
$\begin{array}{l}\text { Horas dedicadas a la } \\
\text { ocupación secundaria }\end{array}$ & 14,53 & 7,36 & 8,03 & - & 6,11 & 6,92 \\
\hline
\end{tabular}

Nota: Elaborado con base en datos de la ENUT 2017. 
En este apartado se ha analizado el ámbito visible de la actividad económica de las jefas y jefes de hogar y se hace referencia también a las jefas que se encuentran solas en la conducción del núcleo familiar. Como conclusión general podemos decir que existen desigualdades en los ingresos, en la posición que ocupan jefas y jefes en sus ocupaciones principales, así como en las horas que dedican a sus ocupaciones remuneradas. Estas diferencias se acentúan en el caso de los hogares monoparentales liderados por mujeres, por lo menos, en algunas de sus categorías.

Esta situación no solo afectará a los individuos que se encuentren en esta posición relacional en el hogar, sino que además afectará al núcleo familiar completo. Un ejemplo es el caso de hijas e hijos que deben trabajar o combinar estudios con la participación en el mercado laboral, lo que ubicará a estas personas en una posición de desigualdad frente a los hogares biparentales.

\section{El ámbito del trabajo invisibilizado}

En la Tabla 4 observamos que el promedio de las horas semanales de trabajo no remunerado de los hombres es menos de la mitad que el de las mujeres al frente del hogar. Además, la media de horas semanales que las mujeres en hogares monoparentales dedican a estas tareas y la media del conjunto de las mujeres no difieren mucho. Las separadas son las que se ubican por encima de la media de las jefas y las viudas son las que más se distancian del dato general (4 horas).

\section{Tabla 4}

Horas semanales de jefas y jefes dedicadas a tareas no remuneradas (media)

\begin{tabular}{lcccccc}
\hline Categoría & Jefes & Jefas & $\begin{array}{l}\text { Jefas } \\
\text { solteras }\end{array}$ & $\begin{array}{l}\text { Jefas } \\
\text { viudas }\end{array}$ & $\begin{array}{l}\text { Jefas } \\
\text { divorciadas }\end{array}$ & $\begin{array}{l}\text { Jefas } \\
\text { separadas }\end{array}$ \\
\hline $\begin{array}{l}\text { Horas dedicadas a tareas } \\
\text { no remuneradas (trabajo } \\
\text { doméstico no remunerado) }\end{array}$ & 16,24 & 39,77 & 37,69 & 34,75 & 35,91 & 42,05 \\
\begin{tabular}{l} 
Horas dedicadas al cuidado \\
\hline
\end{tabular} & 6,82 & 11,12 & 10,94 & 7,95 & 8,05 & 12,00 \\
\hline
\end{tabular}

Nota: Elaborado con base en datos de la ENUT 2017.

La dedicación media de las jefas es mayor en las tareas no remuneradas respecto a los jefes, e incluso es similar a la suma de las medias que estas mujeres dedican a las tareas remuneradas (principal y secundaria): 39,8 horas dedicadas a las tareas no remuneradas frente a 40,9 horas dedicadas a las tareas remuneradas. Podría suponerse una situación de igualdad en la posición filial, es decir, en responsabilidades, pero la carga de trabajo es distinta.

Mientras que en las ocupaciones principales remuneradas los hombres trabajan en promedio 13 horas más que las mujeres (sumando las secundarias trabajan 20 horas más), si sumamos a estas horas las de trabajo no remunerado, la situación se revierte y 
son las mujeres las que trabajan 3,5 horas más que los hombres ${ }^{8}$. Hay una sobrecarga de trabajo para las mujeres en la posición de jefas en relación con los jefes, lo que además supone una mayor contribución a la economía del hogar y de la sociedad, si ampliamos nuestra definición de actividad económica incluyendo las tareas que se realizan fuera del mercado. Es, en definitiva, una posición desigual si además tenemos en cuenta que estas horas no están remuneradas.

Dentro de las tareas que permanecen invisibilizadas encontramos las tareas de cuidado. Si analizamos las horas promedio que los jefes le dedican a la semana vemos, en la Tabla 4 , que dedican 6,82 horas, mientras que las jefas dedican a estas tareas 11,12 horas a la semana, casi el doble. Las horas que las mujeres jefas de familias monoparentales dedican al cuidado también es mayor ${ }^{9}$, si bien, son las separadas y las solteras las que más se acercan a la media, y las que más carga horaria tienen en estas tareas.

Si complementamos esta información con las horas que las compañeras y compañeros dedican al cuidado (Tabla 5), vemos que las compañeras casi doblan las horas a la semana que dedican los cónyuges hombres a las tareas de cuidado. Las mujeres que lideran un hogar solo dedican 2,02 horas menos que las cónyuges.

\section{Tabla 5}

Horas semanales de compañeras y compañeros dedicadas a tareas no remuneradas (media)

\begin{tabular}{lll}
\hline Categoría & Compañeras & Compañeros \\
\hline $\begin{array}{l}\text { Horas a las tareas no remuneradas (excluye } \\
\text { cuidado) }\end{array}$ & 37,89 & 12,19 \\
$\begin{array}{l}\text { Horas a las tareas no remuneradas (trabajo } \\
\text { doméstico no remunerado) }\end{array}$ & 45,93 & 16,02 \\
\begin{tabular}{l} 
Horas dedicadas al cuidado \\
\hline
\end{tabular}
\end{tabular}

Nota: Elaborado con base en datos de la ENUT 2017.

Es posible que en los hogares monoparentales exista una red de contención, es decir, otras familiares que compartan la carga de las tareas domésticas y de cuidado. No obstante, no es el objetivo de este trabajo analizar esta situación. En cuanto al tiempo dedicado a estas tareas, sigue existiendo una carga mayor en las mujeres, independientemente de que estas tengan o no el liderazgo del hogar, lo que supondrá mayores responsabilidades.

8 Según la Tabla 3 las jefas trabajan 33,53 horas en su ocupación principal, mientras que los hombres trabajan 46,4 horas. Si tenemos en cuenta las secundarias las mujeres trabajan 40,88 horas en promedio y 60,93 los hombres, si sumamos a estas últimas cifras el trabajo no remunerado las horas trabajadas son 77,17 horas semanales para los hombres y 80,65 horas semanales para las mujeres.

9 En este caso, reconocemos la dificultad de marcar el límite entre tareas remuneradas y de cuidado, en casos en donde las mujeres llevan a sus hijos al lugar de trabajo (venta ambulante, por ejemplo). Véase Carrasco (2013). 


\section{Tabla 6}

Horas semanales dedicadas a tareas no remuneradas de los jefes y jefas de hogar (media)

\begin{tabular}{|c|c|c|c|c|c|c|}
\hline Tareas & Jefe & Jefa & $\begin{array}{l}\text { Jefas } \\
\text { solteras }\end{array}$ & $\begin{array}{l}\text { Jefas } \\
\text { viudas }\end{array}$ & $\begin{array}{l}\text { Jefas } \\
\text { divorciadas }\end{array}$ & $\begin{array}{l}\text { Jefas } \\
\text { separadas }\end{array}$ \\
\hline $\begin{array}{l}\text { Preparación servicio de } \\
\text { bebidas y alimentos }\end{array}$ & 6,26 & 16,47 & 14,77 & 15,58 & 14,66 & 17,02 \\
\hline $\begin{array}{l}\text { Limpieza y mantenimiento } \\
\text { de vivienda }\end{array}$ & 4,31 & 10,21 & 10,60 & 10,35 & 9,02 & 10,21 \\
\hline Tareas & Jefe & Jefa & $\begin{array}{l}\text { Jefas } \\
\text { solteras }\end{array}$ & $\begin{array}{l}\text { Jefas } \\
\text { viudas }\end{array}$ & $\begin{array}{l}\text { Jefas } \\
\text { divorciadas }\end{array}$ & $\begin{array}{l}\text { Jefas } \\
\text { separadas }\end{array}$ \\
\hline $\begin{array}{l}\text { Construcción y reparaciones } \\
\text { menores de la vivienda y } \\
\text { vehículos }\end{array}$ & 2,62 & 1,47 & 1,51 & 2,14 & 1,39 & 1,10 \\
\hline $\begin{array}{l}\text { Limpieza y cuidado de ropa } \\
\text { y calzado }\end{array}$ & 1,55 & 4,66 & 3,97 & 4,96 & 4,15 & 4,65 \\
\hline
\end{tabular}

Nota: Elaborado con base en datos de la ENUT 2017.

Se observa en la Tabla 6 que las jefas superan a los jefes en horas de dedicación a la semana en todas las tareas domésticas, menos en las tareas de construcción y reparaciones menores en la vivienda y vehículos.

Los datos analizados en este apartado nos sugieren que las tareas no remuneradas están a cargo de las mujeres. Además, las jefas trabajan más que los jefes, si contemplamos el conjunto de las tareas (remuneradas y no remuneradas).

\section{Reflexiones finales}

Los resultados arrojados en el análisis de los datos de la muestra de la ENUT 2017 han revelado que existen desigualdades en los ingresos, en la posición que ocupan jefas y jefes en sus ocupaciones principales, así como en las horas que dedican a sus labores remuneradas. Estas diferencias se acentúan en el caso de los hogares monoparentales liderados por mujeres. Las jefas son quienes realizan la aportación principal al hogar, las familias lideradas por mujeres padecerán una desigualdad de ingresos mayor, que podrán o no compensarse con el ingreso de otro miembro, pero que, en cualquier caso, afecta al conjunto del hogar.

En la mayoría de los hogares jefaturados por mujeres, estas no tienen pareja, lo cual implica que son ellas las responsables del ingreso de recursos al hogar. Estos recursos serán menores a los de hogares regentados por hombres por su posición en el mercado laboral.

En análisis de los datos referentes a las tareas no remuneradas se ha constatado que son las jefas quienes más carga horaria tienen. Además, trabajan más horas en total que los jefes, si se tienen en cuenta también las horas de trabajo remunerado. 
Así, las mujeres a cargo del hogar tendrán las mismas responsabilidades que los hombres, pero con ingresos menores, y con menos tiempo para dedicar al ocio y a su desarrollo personal. No solo ellas se verán afectadas por esta situación, los niños y niñas que vivan en estos hogares padecerán de estas desigualdades de ingresos y de tiempo, ya que, seguramente, tendrán que sustituir parte del trabajo no remunerado que realizan los adultos en los hogares.

Pese a las dificultades que pueden presentar los datos, al no haberse realizado una estimación a nivel nacional, el análisis sugiere la necesidad de implementar políticas públicas que pongan remedio a estas desigualdades tanto a nivel económico como social y personal.

En primer lugar, se deberían contemplar las tareas ocultas que se realizan en el ámbito doméstico como parte intrínseca de la economía productiva, introduciendo, en consecuencia, nuevos indicadores y conceptos para su medición. De esta forma, se presentaría una imagen más correcta sobre la desigualdad que padecen las mujeres.

Algunas de las políticas públicas que pueden aliviar esta realidad son las que permiten una repartición más justa de la responsabilidad en las tareas no remuneradas. Por ejemplo, la creación de permisos de maternidad y paternidad iguales e intransferibles, lo que contribuiría a la igualdad en términos de tiempos dedicados a las tareas no remuneradas, pero también a los tiempos dedicados a las tareas remuneradas. Es una medida que contribuye a la igualdad de oportunidades en el mercado laboral, a la que debería sumarse la obligación de que hombres y mujeres percibieran salarios iguales por la realización de las mismas tareas.

Desde la perspectiva de las políticas activas de empleo, la oferta de cursos que contribuyan a mejorar el acceso al mercado de trabajo, a través de una mayor la formación y cualificación. Estas políticas deben tener en cuenta la situación especial de aquellas mujeres que no tienen red de contención en sus hogares para el cuidado de niños y personas dependientes.

El hecho de que las jefas de hogar dediquen más tiempo al trabajo (remunerado más no remunerado) tiene implicaciones en sus propias vidas (al no disponer del mismo tiempo de ocio o para dedicar a otras actividades) pero también en la vida de quienes componen estos hogares. Si bien existen ayudas para los niños y niñas en situación de vulnerabilidad, como las otorgadas por el plan "Crecemos" del Instituto Mixto de Ayuda Social, son necesarios apoyos que se dirijan especialmente a los hogares monoparentales, adecuándose específicamente a las necesidades concretas de estos hogares, que pueden incluir la problemática del uso del tiempo y de la ausencia de redes sociales de contención. 
Un ejemplo es la creación de espacios donde se pueda compartir el cuidado, que creen redes de apoyo mutuo vinculados al barrio o territorio, impulsados por los gobiernos locales, mediante la cesión de espacios y subvenciones ${ }^{10}$.

También es requerida una mayor inversión pública en educación infantil que contemple la acogida de niñas y niños entre 0 y 3 años. Esta medida podría complementarse con una mejor adaptación de los horarios laborales y los horarios de los centros educativos. También se podría ampliar la inversión pública para aumentar los apoyos a personas dependientes, a través de, por ejemplo, centros de cuidado público.

En la aplicación de estas políticas, se debería tener en cuenta la existencia de una mayor condición de vulnerabilidad en el colectivo de mujeres solas al frente del hogar. Es necesaria la existencia de políticas que garanticen el acceso al mercado laboral y a unos ingresos dignos para este colectivo, así como una red de contención ante las necesidades que este tipo de hogares puede presentar.

Por último, consideramos que es preciso aclarar que estas políticas solo suavizarían las desigualdades existentes entre hombres y mujeres, tanto en la percepción de ingresos como en las condiciones sociales y personales, ya que mientras el eje del sistema económico se ubique en el beneficio y el rol de las mujeres siga asociándose de forma artificial a los cuidados no será posible erradicarlas. Es necesario un cambio de eje que ubique a la vida en el centro de la economía y la política, y poner de relieve la incapacidad del sistema económico actual para garantizar las condiciones de vida de todas las personas. Es urgente promover cambios culturales y sociales que contribuyan a incorporar los múltiples aspectos que conforman la identidad de las personas, además de eliminar la normatividad que liga los aspectos de esta identidad a determinados roles y estigmas.

10 Ver ejemplos de estas medidas en "Economía Feminista "72 propuestas"" 


\section{Bibliografía}

Barrientos Esquivel, A., Fonseca Sánchez, P., Mora Steiner, S., \& Valverde Chinchilla, R. (2013). Análisis de las transformaciones en la estructura y conformación de los hogares y familias en Costa Rica a la luz de los Censos de Población y Vivienda 2000-2011. http://repositorio. sibdi.ucr.ac.cr:8080/xmlui/bitstream/handle/123456789/1624/35281.pdf?sequence=1

Barrón López, S. (2002). Familias monoparentales: Un ejercicio de clarificación conceptual y sociológica. Revista del Ministerio de Trabajo y Asuntos Sociales 40, 13-30

Benería, L. (1981). Reproducción, producción y división sexual del trabajo. Mientras tanto, (6), 47-84. Icaria.

Brunet Icart, I., \& Santamaría Velasco, C. A. (2016). La economía feminista y la división sexual del trabajo. Culturales, 4(1), 61-86. http://www.scielo.org.mx/pdf/cultural/v4n1/2448-539X-cultural-4-01-00061.pdf

Carrasco, C. (2001). La sostenibilidad de la vida humana: ¿Un asunto de mujeres? Mientras tanto, $82,43-70$. Icaria

Carrasco, C. (2013). El cuidado como eje vertebrador de una nueva economía. Cuadernos de relaciones laborales, 31(1), 39-56.

Carrasco, C. (2017). La economía feminista. Un recorrido a través del concepto de reproducción. Ekonomiaz, 91(01), 50-75.

INEC. (2017). Encuesta Nacional de Uso del Tiempo 2017. Resultados Generales. 18/09/2019 https://www.inec.go.cr/sites/default/files/documetos-biblioteca-virtual/reenut2017.pdf

OIT. (2019a). Panorama laboral temático (5). Mujeres en el mundo del trabajo: Retos pendientes hacia una efectiva equidad en América Latina y el Caribe. https://www.ilo.org/wcmsp5/ groups/public/---americas/---ro-lima/documents/publication/wcms_715183.pdf

OIT. (2019b). Informe Mundial sobre Salarios 2018 / 2019. Qué hay detrás de la brecha salarial de género. https://www.ilo.org/wcmsp5/groups/public/---dgreports/---dcomm/---publ/ documents/publication/wcms_650653.pdf

Pérez Orozco, A. (2014). Subversión feminista de la economía: Aportes para un debate sobre el conflicto capital-vida. Traficantes de sueños. 